\title{
Both moderate and severe exacerbations accelerate physical activity decline in COPD patients
}

\author{
To the Editor:
}

Physical activity (PA) is a relevant outcome measure in chronic obstructive pulmonary disease (COPD). Low PA is prevalent and drives prognosis [1]. Unfortunately, the determinants of PA and its change over time are poorly understood [1]. The fact that the PA progressively declines over time along with worsening of lung function and health status [2] suggests that the PA decline could be due to the progression of the disease and specifically to acute exacerbations [3]. An acute reduction in PA at the onset has been reported both in severe exacerbations requiring a hospitalisation [4] and in ambulatory treated exacerbations [5, 6]. A sustained PA reduction has been shown 1 month after hospital discharge [4] whereas PA almost returns to stable levels after community-treated exacerbations [6]. One study found faster PA decline in patients with a history of two or more exacerbations in the 12 months prior to the study [5]. This analysis did not adjust for confounders of the association (e.g. airflow obstruction, symptom burden) or external variables influencing PA (e.g. climate). It could also be argued that the greater decline in PA was due to lower health status at baseline. Although PA is an important outcome for COPD patients, little is known about the role of exacerbations in patients' experience of PA. Importantly, both the amount of activity and difficulties experienced during activity are integral to the concept of PA limitation [7]. The aim of the present analysis was to assess the association between the number and severity of exacerbations and changes in PA and PA experience.

As part of the IMI-JU PROactive study [7, 8], patients with COPD from mixed healthcare settings (tertiary hospitals, rehabilitation centres and primary care) in five European centres (Athens, Greece; Edinburgh and London, UK; Leuven, Belgium; and Groningen, The Netherlands) were studied at baseline and at 12 months' follow-up. Patients followed and those lost to follow-up were similar. Objective PA was measured using the Dynaport Movemonitor accelerometer (McRoberts BV, The Hague, The Netherlands). The main PA outcome analysed was the change (difference between baseline and 1 year) in daily step count; the secondary outcome was the change in intensity during locomotion. Patient experience of PA was assessed with the Clinical visit PROactive Physical Activity in COPD (C-PPAC) instrument [7], which consists of a total score and amount and difficulty scores. The number and severity of COPD exacerbations during the 12-month follow-up was retrieved [8]. We defined exacerbations as moderate (treated in an ambulatory setting with systemic antibiotics and/or systemic glucocorticosteroids) or severe (requiring hospital admission), following the GOLD initiative guidelines [9]. In addition to the number of exacerbations, we generated a variable combining the number and the severity of exacerbations. The association between exacerbation variables and PA change was analysed using regression analysis adjusted for baseline PA values. To remove the effect of potential confounders (listed in the caption of figure 1), we built a multivariable linear model for each PA outcome. Sensitivity analyses were performed excluding patients 1) with a COPD admission in the year before recruitment, 2 ) with extreme PA values ( $\leqslant 5$ th or $\geqslant 95$ th percentiles) and 3 ) with a COPD admission 2 months prior to the final measurement to exclude an acute effect. As post hoc explanatory analyses we tested the (bivariate) association between exacerbation variables and change in forced expiratory volume in $1 \mathrm{~s}$ (FEV1), 6-min walk distance (6MWD) and modified Medical Research Council (mMRC) score, adjusted for baseline. All statistical analyses were performed using SAS 9.4 (SAS Institute Inc., Cary, NC, USA).

The present analysis is based on 141 patients with valid data at baseline and follow-up (75\% male, with a mean \pm SD age of $67 \pm 8$ years, BMI $27 \pm 5 \mathrm{~kg} \cdot \mathrm{m}^{-2}, \mathrm{FEV} 159 \pm 21 \%$ predicted) of whom 81 (57\%) experienced

@ERSpublications

Exacerbations have a negative impact on long-term daily physical activity of COPD patients http://ow. ly/olGl30hkdMl

Cite this article as: Demeyer $\mathrm{H}$, Costilla-Frias $\mathrm{M}$, Louvaris $\mathrm{Z}$, et al. Both moderate and severe exacerbations accelerate physical activity decline in COPD patients. Eur Respir J 2018; 51: 1702110 [https://doi.org/10.1183/13993003.02110-2017]. 

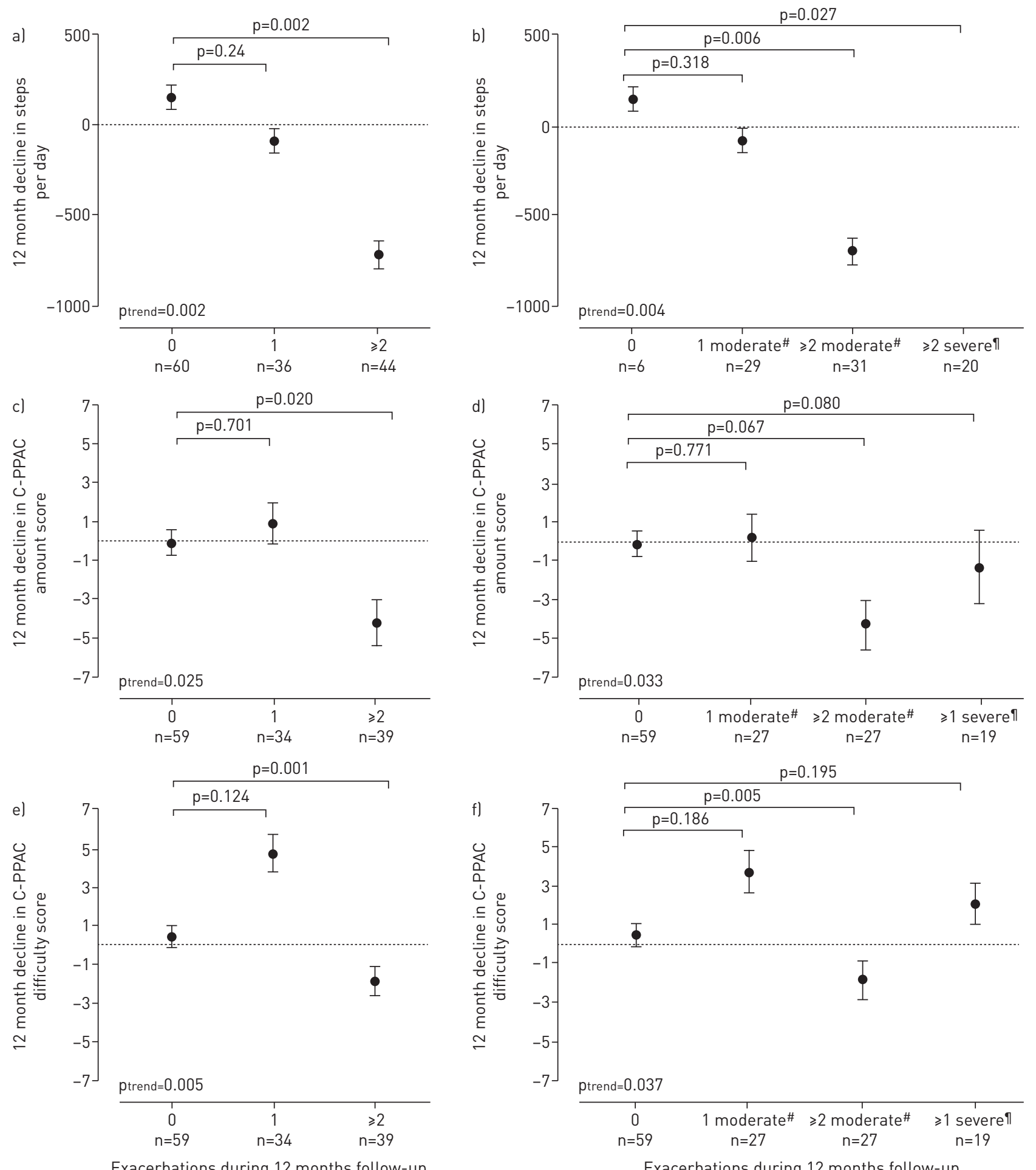

Exacerbations during 12 months follow-up

FIGURE 1 Decline in step count (a, b) and Clinical visit PROactive Physical Activity in COPD (C-PPAC) instrument score (c- $f$ ) according to chronic obstructive pulmonary disease (COPD) exacerbations in the 12-month follow-up (multivariable linear regression). Data are presented as estimated marginal means (least squares means) and SEM. Final models are adjusted for baseline physical activity and total COPD assessment test (CAT) score. Other potential confounders lage, gender, smoking habit, body mass index, any COPD admissions in previous 12 months, forced expiratory volume in $1 \mathrm{~s}\left(\mathrm{FEV}_{1}\right) \%$ predicted, forced vital capacity (FVC) \% predicted, FEV $1 / F V C$ ratio, American Thoracic Society/European Respiratory Society stage, 6-min walking distance, modified Medical Research Council score and Hospital Anxiety and Depression Scale scores) were tested and finally not included because they were not independently related to both the exposure and the outcome, nor modified (>10\% change) the estimates for the remaining variables. " : patients without severe exacerbations; " irrespective of the number of moderate exacerbations. 
at least one exacerbation during follow-up. In the whole group there was a small, nonsignificant decline in step count (5209 \pm 3050 steps per day at baseline versus $5019 \pm 3099$ steps per day at follow-up), while movement intensity $\left(1.82 \pm 0.26 \mathrm{~m} \cdot \mathrm{s}^{-2}\right.$ at baseline versus $1.80 \pm 0.28 \mathrm{~m} \cdot \mathrm{s}^{-2}$ at follow-up), exercise capacity $(452 \pm 119 \mathrm{~m}$ at baseline versus $450 \pm 123 \mathrm{~m}$ at follow-up) and FEV1 (1.62 $\pm 0.69 \mathrm{~L}$ at baseline versus $1.63 \pm 0.69 \mathrm{~L}$ at follow-up) did not change. Patients had a baseline mean C-PPAC total score of $69 \pm 13$, with domain scores for amount and difficulty $64 \pm 16$ and $74 \pm 15$, respectively.

The number of exacerbations was related to the decline in step count. In the multivariable model, a mean \pm SE change of $251 \pm 207,-144 \pm 262$ and $-797 \pm 244$ steps per day was observed in patients who had presented no, one, or two or more exacerbations during follow-up respectively (figure 1a). Patients experiencing two or more moderate exacerbations ( -753 steps per day) and those experiencing at least one severe exacerbation ( -705 steps per day) showed a larger decline in PA than those with no exacerbations (figure 1b). There was no association between exacerbations during follow-up and changes in PA intensity $\left(\mathrm{m} \cdot \mathrm{s}^{-2}\right)$. In relation to patient experience of PA, figure $1 \mathrm{c}$ and $\mathrm{e}$ show that patients with two or more exacerbations suffered more deterioration in C-PPAC amount ( -4.2 points) and difficulty ( -1.9 points) scores than patients having no or only one exacerbation. Patients experiencing two or more moderate exacerbations or at least one severe exacerbation showed a larger decline in C-PPAC amount and difficulty scores than those with no exacerbations; however, some of these associations were not statistically significant (figure 1d and f). All sensitivity analyses yielded similar results. Having exacerbations was related to a significant $(\mathrm{p}<0.01)$ larger increase in mMRC score but not to changes in $6 \mathrm{MWD}$ or FEV1.

The present analyses show that the acute drop in PA after an exacerbation [4-6] has an important and lasting effect that cannot be attributed to confounders or baseline PA. A higher exacerbation frequency was, as expected, associated with a more pronounced PA decline. Patients with frequent exacerbations constitute a specific disease phenotype with a worse prognosis, specifically a faster loss in lung function [10], a greater worsening of health status [11] and a substantial reduction in the amount of PA ( -797 steps per day) as indicated by our findings. An unexpected finding from this study is that two or more moderate events result in a long-term decline that is clinically relevant [12] and equivalent to that of a severe event, confirming the importance of prevention and early management of exacerbations regardless of the severity [9].

The fact that exacerbations result in a decrease in the amount of PA can be interpreted as part of the multiple systemic consequences [9]. First, we could hypothesise that the decline is a consequence of a loss in exercise capacity. Against this hypothesis, our data fail to show an association between exacerbations during follow-up and changes in exercise capacity. Second, the PA decline could potentially be explained based on a worsening of symptoms during an exacerbation leading to more inactivity, bringing patients in a vicious cycle of symptoms and inactivity [13]. Based on our explanatory analysis showing an association between exacerbations and an increase in dyspnoea, the latter hypothesis would be a plausible mechanism.

The present results have important implications for daily clinical practice and underscore the importance of identifying frequent exacerbators, as supported by the combined GOLD classification [9]. Our results support the promotion of interventions to increase PA, such as coaching programmes in the less [14] or pulmonary rehabilitation in the more severe patients [15] after an exacerbation.

Heleen Demeyer 1,2,3,4,5,17, Marcos Costilla-Frias ${ }^{3,6,17}$, Zafeiris Louvaris ${ }^{1,7}$, Elena Gimeno-Santos $\oplus^{2,3,4,8}$, Maggie Tabberer', Roberto A. Rabinovich ${ }^{10}$, Corina de Jong ${ }^{11,12}$, Michael I. Polkey ${ }^{13}$, Nicholas S. Hopkinson $\odot^{13}$, Niklas Karlsson $^{14}$, Ignasi Serra ${ }^{2,3,4}$, Ioannis Vogiatzis ${ }^{7,15}$, Thierry Troosters ${ }^{1,5}$ and Judith Garcia-Aymerich $\circledast^{2,3,4}$ on behalf of the PROactive Consortium

${ }^{1} \mathrm{KU}$ Leuven, Dept of Rehabilitation Sciences, Leuven, Belgium. ${ }^{2}$ ISGlobal, Center for Research in Environmental Epidemiology (CREAL), Barcelona, Spain. ${ }^{3}$ Universitat Pompeu Fabra (UPF), Barcelona, Spain. ${ }^{4}$ CIBER Epidemiología y Salud Pública (CIBERESP), Barcelona, Spain. ${ }^{5}$ University Hospitals Leuven, Dept of Respiratory Diseases, Leuven, Belgium. ${ }^{6}$ Universitat Autònoma de Barcelona (UAB), Barcelona, Spain. ${ }^{7}$ Faculty of Physical Education and Sports Sciences, National and Kapodistrian University of Athens, Athens, Greece. ${ }^{8}$ Institut d'Investigacions Biomèdiques August Pi i Sunyer (IDIBAPS), Hospital Clínic de Barcelona, Pneumology Dept, Barcelona, Spain. ${ }^{9}$ GlaxoSmithKline, Uxbridge, UK. ${ }^{10} \mathrm{ELEGI} / \mathrm{Colt}$ Laboratory, UoE/MRC Centre for Inflammation Research, The University of Edinburgh, Edinburgh, UK. ${ }^{11}$ GRIAC-Primary Care, Dept of General Practice and Elderly Care, University of Groningen, University Medical Center Groningen (UMCG), Groningen, The Netherlands. ${ }^{12}$ Groningen Research Institute for Asthma and COPD (GRIAC), University of Groningen, University Medical Center Groningen (UMCG), Groningen, The Netherlands. ${ }^{13}$ Royal Brompton and Harefield NHS Foundation Trust and Imperial College, NIHR Respiratory Biomedical Research Unit, London, UK. ${ }^{14}$ AstraZeneca, Gothenburg, Sweden. ${ }^{15}$ Dept of Sport, Exercise and Rehabilitation, Faculty of Health and Life Sciences, Northumbria University, Newcastle, UK. ${ }^{16} \mathrm{~A}$ full list of the PROactive consortium members and their affiliations can be found in the Acknowledgements section. ${ }^{17}$ Both authors contributed equally.

Correspondence: Judith Garcia-Aymerich, ISGlobal, Doctor Aiguader 88, 08003 Barcelona, Spain.

E-mail: Judith.garcia@isglobal.org

Received: June 232017 | Accepted after revision: Oct 272017 
Acknowledgements: We are grateful for the input from the PROactive project ethics board, advisory board and patient input platform. The PROactive Consortium members are as follows: Nathalie Ivanoff (Almirall, Barcelona, Spain); Niklas Karlsson and Solange Corriol-Rohou (AstraZeneca AB, Gothenburg, Sweden); Ian Jarrod (British Lung Foundation, London, UK); Damijen Erzen (Boehringer Ingelheim, Nieder-Ingelheim, Germany); Mario Scuri and Roberta Montacchini (Chiesi Farmaceutici S.A., Parma, Italy); Paul McBride (Choice Healthcare Solutions, Hitchin, UK); Nadia Kamel (European Respiratory Society, Lausanne, Switzerland); Maggie Tabberer (GlaxoSmithKline, Uxbridge, UK); Thierry Troosters, Wim Janssens, Heleen Demeyer and Fabienne Dobbels (Katholieke Universiteit Leuven, Leuven, Belgium); Judith Garcia-Aymerich (ISGlobal, Center for Research in Environmental Epidemiology (CREAL), Barcelona, Spain); Pim de Boer (Netherlands Lung Foundation, Amersfoort, The Netherlands); Karoly Kulich (Novartis, Basel, Switzerland); Michael I. Polkey and Nick S. Hopkinson (Royal Brompton and Harefield NHS Foundation Trust, London, UK); Ioannis Vogiatzis (Thorax Research Foundation, Athens, Greece); Enkeleida Nikai (UCB, Brussels, Belgium); Thys van der Molen and Corina De Jong (University Medical Center, Groningen, The Netherlands); Roberto A. Rabinovich and Bill MacNee (University of Edinburgh, Edinburgh, UK); Milo A. Puhan and Anja Frei (University of Zurich, Zurich, Switzerland).

Support statement: The PROactive project is funded by the Innovative Medicines Initiative Joint Undertaking (IMU JU) \#115011. This work was supported by the Flemish Research Foundation (grant \# G.0871.13). H. Demeyer is the recipient of a joint ERS/SEPAR Fellowship (LTRF 2015) and is a post-doctoral research fellow of the FWO-Flanders. Z. Louvaris is the recipient of an ERS fellowship (LTRF 2016) and is a post-doctoral research fellow of the FWO-Flanders. ISGlobal is a member of the CERCA Programme, Generalitat de Catalunya. Funding information for this article has been deposited with the Crossref Funder Registry.

Conflict of interest: Disclosures can be found alongside this article at erj.ersjournals.com

\section{References}

1 Gimeno-Santos E, Frei A, Steurer-Stey C, et al. Determinants and outcomes of physical activity in patients with COPD: a systematic review. Thorax 2014; 69: 731-739.

2 Waschki B, Kirsten AM, Holz O, et al. Disease progression and changes in physical activity in patients with chronic obstructive pulmonary disease. Am J Respir Crit Care Med 2015; 192: 295-306.

3 Troosters T, Demeyer H. Physical inactivity as a missing link in understanding the progression of chronic obstructive pulmonary disease. Am J Respir Crit Care Med 2015; 192: 267-269.

4 Pitta F, Troosters T, Probst VS, et al. Physical activity and hospitalization for exacerbation of COPD. Chest 2006; 129: 536-544.

5 Alahmari AD, Patel AR, Kowlessar BS, et al. Daily activity during stability and exacerbation of chronic obstructive pulmonary disease. BMC Pulm Med 2014; 14: 98.

6 Ehsan $\mathrm{M}$, Khan $\mathrm{R}$, Wakefield $\mathrm{D}$, et al. A longitudinal study evaluating the effect of exacerbations on physical activity in patients with chronic obstructive pulmonary disease. Ann Am Thorac Soc 2013; 10: 559-564.

7 Gimeno-Santos E, Raste Y, Demeyer H, et al. The PROactive instruments to measure physical activity in patients with chronic obstructive pulmonary disease. Eur Respir J 2015; 46: 988-1000.

8 Demeyer H, Gimeno-Santos E, Rabinovich RA, et al. Physical activity characteristics across GOLD quadrants depend on the questionnaire used. PLoS One 2016; 11: e0151255.

9 Global Strategy for the Diagnosis, Management and Prevention of COPD. Global Initiative for Chronic Obstructive Lung Disease (GOLD) 2017; Available from: www.goldcopd.org

10 Donaldson GC, Seemungal TA, Bhowmik A, et al. Relationship between exacerbation frequency and lung function decline in chronic obstructive pulmonary disease. Thorax 2002; 57: 847-852.

11 Miravitlles M, Ferrer M, Pont A, et al. Effect of exacerbations on quality of life in patients with chronic obstructive pulmonary disease: a 2 year follow up study. Thorax 2004; 59: 387-395.

12 Demeyer H, Burtin C, Hornikx M, et al. The minimal important difference in physical activity in patients with COPD. PLoS One 2016; 11: e0154587.

13 Troosters T, Van der Molen T, Polkey M, et al. Improving physical activity in COPD: towards a new paradigm. Respir Res 2013; 14: 115.

14 Demeyer H, Louvaris Z, Frei A, et al. Physical activity is increased by a 12-week semiautomated telecoaching programme in patients with COPD: a multicentre randomised controlled trial. Thorax 2017; 72: 415-423.

15 Puhan MA, Gimeno-Santos E, Cates CJ, et al. Pulmonary rehabilitation following exacerbations of chronic obstructive pulmonary disease. Cochrane Database Syst Rev 2016; 12: CD005305. 